Delft University of Technology

\title{
A Comparison Between Representative 3D Faces Based on Bi- and Multi-variate and Shape Based Analysis
}

Goto, Lyè; Huysmans, Toon; Lee, Wonsup; Molenbroek, Johan F. M.; Goossens, Richard

DOI

10.1007/978-3-319-96071-5_137

Publication date

2019

Document Version

Final published version

Published in

Proceedings of the 20th Congress of the International Ergonomics Association (IEA 2018)- Volume VII

\section{Citation (APA)}

Goto, L., Huysmans, T., Lee, W., Molenbroek, J. F. M., \& Goossens, R. (2019). A Comparison Between Representative 3D Faces Based on Bi- and Multi-variate and Shape Based Analysis. In S. Bagnara, R.

Tartaglia, S. Albolino, T. Alexander, \& Y. Fujita (Eds.), Proceedings of the 20th Congress of the International Ergonomics Association (IEA 2018)- Volume VII: Ergonomics in Design, Design for All, Activity Theories for Work Analysis and Design, Affective Design (Vol. VII, pp. 1355-1364). (Advances in Intelligent Systems and Computing; Vol. 824). Springer Science+Business Media. https://doi.org/10.1007/978-3-319-96071-5_137

Important note

To cite this publication, please use the final published version (if applicable).

Please check the document version above.

\section{Copyright}

Other than for strictly personal use, it is not permitted to download, forward or distribute the text or part of it, without the consent of the author(s) and/or copyright holder(s), unless the work is under an open content license such as Creative Commons.

\section{Takedown policy}

Please contact us and provide details if you believe this document breaches copyrights.

We will remove access to the work immediately and investigate your claim. 
Green Open Access added to TU Delft Institutional Repository 'You share, we take care!' - Taverne project

\section{https://www.openaccess.nl/en/you-share-we-take-care}

Otherwise as indicated in the copyright section: the publisher is the copyright holder of this work and the author uses the Dutch legislation to make this work public. 


\title{
A Comparison Between Representative 3D Faces Based on Bi- and Multi-variate and Shape Based Analysis
}

\author{
Lyè Goto $^{1(\bowtie)}(\mathbb{D})$, Toon Huysmans ${ }^{1,2}(\mathbb{D})$, Wonsup Lee $^{3}(\mathbb{D})$, \\ Johan F. M. Molenbroek ${ }^{1}$ (D), and Richard H. M. Goossens ${ }^{1}$ \\ ${ }^{1}$ University of Technology, 2628 CE Delft, The Netherlands \\ L.Goto@TUDelft.nl \\ 2 imec-Vision Lab, Department of Physics, University of Antwerp, \\ Universiteitsplein 1, 2610 Antwerp, Belgium \\ ${ }^{3}$ Handong Global University, Pohang, Gyeongbuk 37554, South Korea
}

\begin{abstract}
In Ergonomic product design, designers need to translate anthropometric data of the target population into product dimensions or sizing systems. Currently, sizing systems are often based on traditional anthropometric data and generally use the variation of one or two key body dimensions directly related to the product. For products that need to closely fit a certain part of the body it is relevant to incorporate multiple key dimensions. This can be realized by a multivariate approach such as a Principal Component Analysis. Over the past decades, there has been an increase in incorporating 3D imaging in anthropometric surveys. In order to integrate the use of 3D anthropometry in product sizing, representative models are used to visualize the variability of the target population. For the development of a ventilation mask for children, this study compares representative models of $3 \mathrm{D}$ faces based on a bivariate, multivariate and shape based analysis of 303 children's faces.
\end{abstract}

Keywords: 3D anthropometry $\cdot$ Children $\cdot$ Ventilation mask Product sizing $\cdot$ Design

\section{Introduction}

One of the challenges a designer faces when designing a product for people where a good fit is essential, is translating anthropometric data of the target population into product dimensions or a sizing system. Currently, sizing systems are often based on traditional anthropometric data and generally use the variation of one or two key body dimensions directly related to the product $[1,2]$. This information is usually presented in tables or scatterplots but rarely represents the complexity of the human form.

For products that need to closely fit a certain part of the body it is relevant to incorporate multiple key dimensions. This can be realized by a multi variate approach which maps the variation in these dimensions in order to generate a sizing system. The most common approach is conducting a principal component analysis (PCA). A PCA is often used to analyse anthropometric datasets in order to discover trends in the variation of multiple dimensions [2-4]. 
Recent developments in the field of 3D imaging have resulted in an increase in the incorporation of $3 \mathrm{D}$ scans in anthropometric surveys $[5,6]$. The richness of $3 \mathrm{D}$ data makes it possible to visualize complex results from anthropometric analysis. By generating 3D models, that represent the variability of the target population, this information becomes more understandable for designers [7]. In addition, designers can then easily utilize these sets of 3D models in their design process, especially with the use of computer aided design (CAD) programs.

In order to develop a ventilation mask for children, this study compares representative models of 3D faces based on bivariate, multivariate and shape based analysis of 303 children's faces, aged 0.5 to 7 years. These results are discussed with regard to the implication for the sizing of the mask and more generally the added value of these methods for designers.

\section{Method}

\subsection{Children's Heads and Face Dataset}

The analysis was based on the existing dataset of 303 children's head and faces. The dataset was acquired by combining traditional and 3D scan extracted anthropometric information [8]. Participants were Dutch children aged from 6 months to 7 years old and included 174 males and 128 females of mixed ethnicity. The heads and faces of the children were captured from four angles (front, $45^{\circ}$ to the left and right, and the back) using a 3dMD face scanner (3dMD Ltd., London UK). The scans were subsequently combined into a 3D scan of the complete head with Artec Studio 9 software (Artec group, Inc., Luxemburg). 19 anthropometric landmarks were defined on the scan and based on these landmarks, 13 facial dimensions were extracted (Fig. 1) by calculating the Euclidian distances between the landmarks (MATLAB $\left.{ }^{\mathrm{TM}}\right)$.
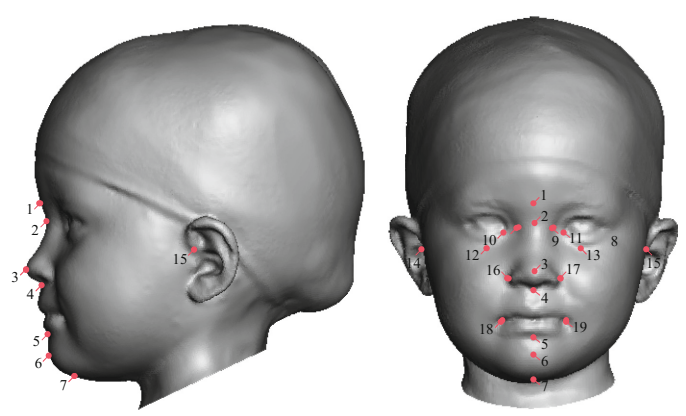

Fig. 1. The landmarks defined on the 3D scan; 1. glabella 2. sellion 3. pronasale 4. subnasale 5 . Sublabiale 6. Pogonion 7. Menton 8/9. Nasal root point (right/left) 10/11. Endocanthion (right/left) 12/13. Infraorbitale (right/left) 14/15. Tragion (right/left) 16/17. Alare (right/left) 18/19. Cheilion (right/left) [8]. 


\subsection{Template Registration}

In order to develop representative models based on the sample, the individual 3D scans needed to be registered. This means that each 3D scan needs to have the same number of vertices at corresponding anatomical locations and a consistent mesh topology. The 3D head scans were registered by using a hybrid template registration method. The template registration was conducted on the face area only. For a more detailed explanation of the method please refer to Lee et al. [9].

\subsection{Development of Face Models with Mask Contours}

First, an average face was generated based on the complete set of template registered models (Fig. 2). Next, a mask contour was projected on the average face as shown in Fig. 1. The contour was drawn through the sellion and promentale landmark and passed both the left and right chellion landmark laterally with a $10 \mathrm{~mm}$ distance to give an approximation of the contact area of the face and mask. Finally, the contour was projected on each individual registered template through vertex correspondence.
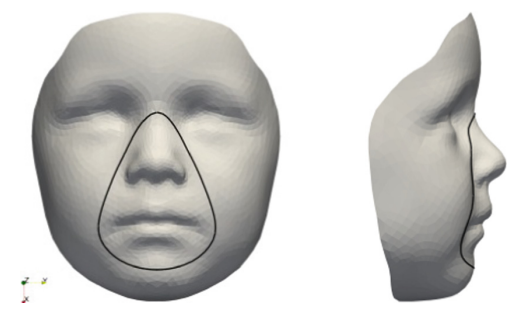

Fig. 2. The template-registered mean face model with the mask contour projection

\subsection{Statistical Analysis of Key Dimensions}

Traditionally, a sizing system is based on one or two relevant key dimensions of the targeted population that are directly related to product dimensions. In previous research, face length and mouth width were considered to be related to the fit of a medical aerosol mask for children [10]. A scatterplot was generated based on sellionpromentale length and mouth width in order analyse the distribution.

Multivariate approaches in sizing system development are often used to incorporate more than two relevant dimensions in order to get an even more representative distribution of the data. A principal component analysis was performed on 9 facial dimensions that were considered relevant to mask design based on previous research namely, face length, sellion-promentale length, nose bridge length, mouth width, nose protrusion, nasal root breadth, nose breadth, interpupillary distance and face width [4, $10,11]$. A scatterplot was generated based on the scores for the first and second principal component. The component scores were calculated as follows: 
PC1 $=0.623 \times($ face width $)+0.907 \times($ face length $)+0.852 \times$ (sellion-promentale length $)+0.818 \times$ (inter-pupillary distance $)+0.636 \times$ (nasal root width $)+0.773 \times$ (nasal bridge length $)+0.754 \mathrm{x}$ (nasal tip protrusion $)+0.728 \mathrm{x}$ (nose width $)+0.647 \mathrm{x}$ (width of mouth).

PC2 $=0.312 \times$ (face width $)+-0.323 \times$ (face length $)+-0.388 \times$ (sellionpromentale length) $+0.289 \times$ (inter-pupillary distance) $+0.466 \mathrm{x}$ (nasal root width $)+-0.459 \mathrm{x}$ (nasal bridge length) $+-0.188 \mathrm{x}$ (nasal tip protrusion $)+0.165 \mathrm{x}$ (nose width) $+0.411 \times$ (width of mouth).

\subsection{Shape Based Analysis}

A principal component analysis was conducted on the face mask area in order to develop shape based RFM's. The face mask area is the area of the template registered face that falls within the mask contour. The principal component analysis was conducted on the vertices of the mesh of the face mask area. The first and second principal component scores were extracted and plotted. The results of the shape based PCA are visualised in Fig. 3.
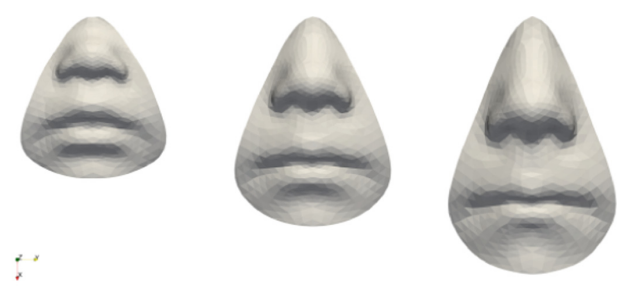

(a) $\mathrm{PC} 1$
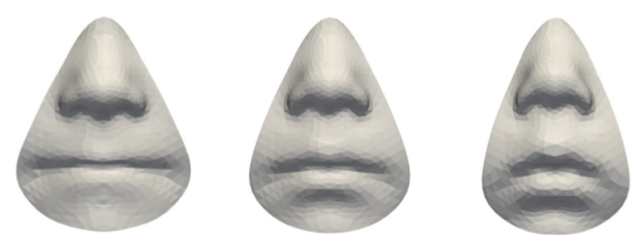

(b) $\mathrm{PC} 2$

Fig. 3. Visualization of the first two principal components showing the average (middle), -3 (left) and +3 (right) standard deviations of the respective PC.

\subsection{Clustering and RFM Development}

Clustering methods have been used to determine product sizes in previous work [10, 12, 13]. A K-means clustering method was utilized (SPSS version 22) in order to divide the sample into four clusters that represent the variation of the childrens faces. This was both done for the bivariate and the multivariate distribution. For the bivariate distribution, the input variables were sellion-promentale length and mouth width. The input variables for the multivariate and shape based distribution were the first and second 
principal component scores of the PCA. Finally, mean representative face models were generated for each cluster for each method (Python and Visualization toolkit (VTK), Kitware Inc.).

\subsection{RFM Comparison}

In order to analyse the representativeness of each RFM for each cluster and per method, the mean distance between the vertices of the RFM and all the members within the same cluster was calculated and subsequently visualized.

\section{Results}

\subsection{K Means Clustering and RFM's}

The bivariate, multivariate and shape based distributions are shown in Figs. 4, 5 and 6. Within each distribution the clusters are indicated and the RFM's are displayed. Tables 1, 2 and 3 show the number of participants assigned to each cluster per method.

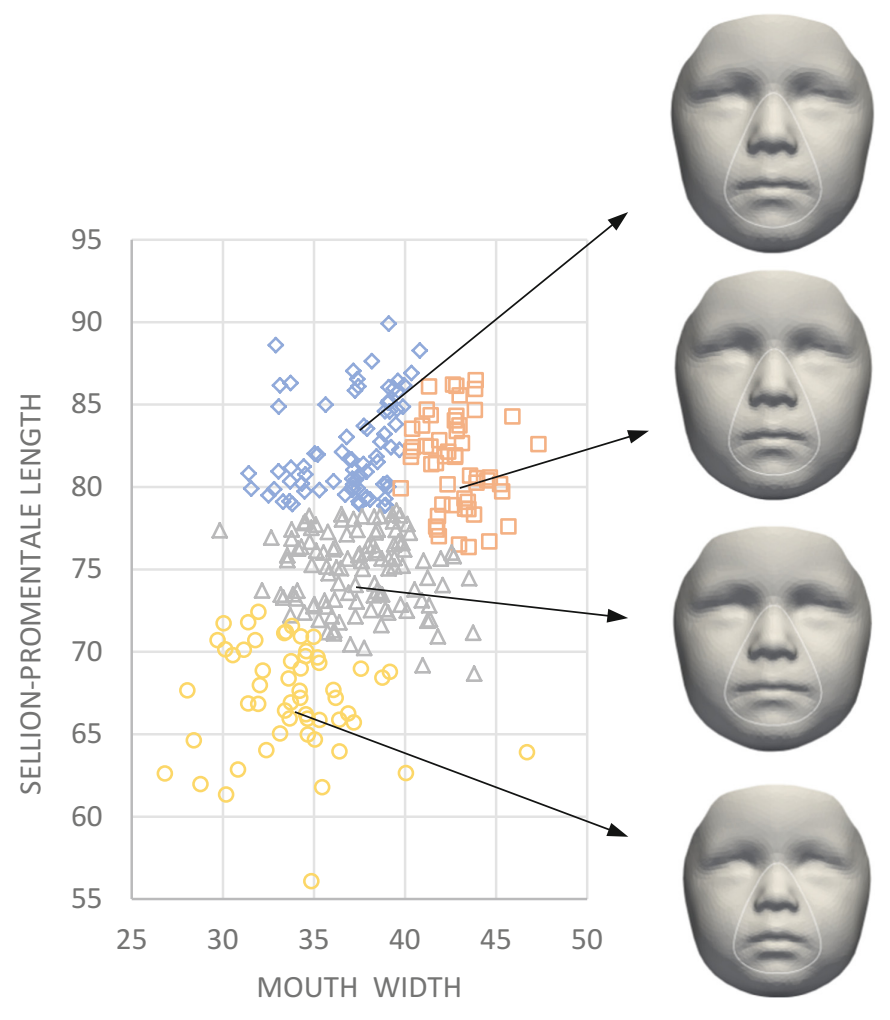

$\diamond$ Cluster $1 \square$ Cluster $2 \Delta$ Cluster $3 \circ$ Cluster 4

Fig. 4. Scatterplot of Mouth Width against Sellion-Promentale length divided into four clusters. 
Table 1. Number of participants in each cluster based on two key dimensions.

\begin{tabular}{l|l|r}
\hline \multicolumn{3}{l}{$\begin{array}{l}\text { Number of cases in } \\
\text { each cluster }\end{array}$} \\
\hline Cluster & 1 & 77 \\
\cline { 2 - 3 } & 2 & 54 \\
\cline { 2 - 3 } & 3 & 115 \\
\cline { 2 - 3 } & 4 & 55 \\
\cline { 2 - 3 } & Total & 301 \\
\hline
\end{tabular}
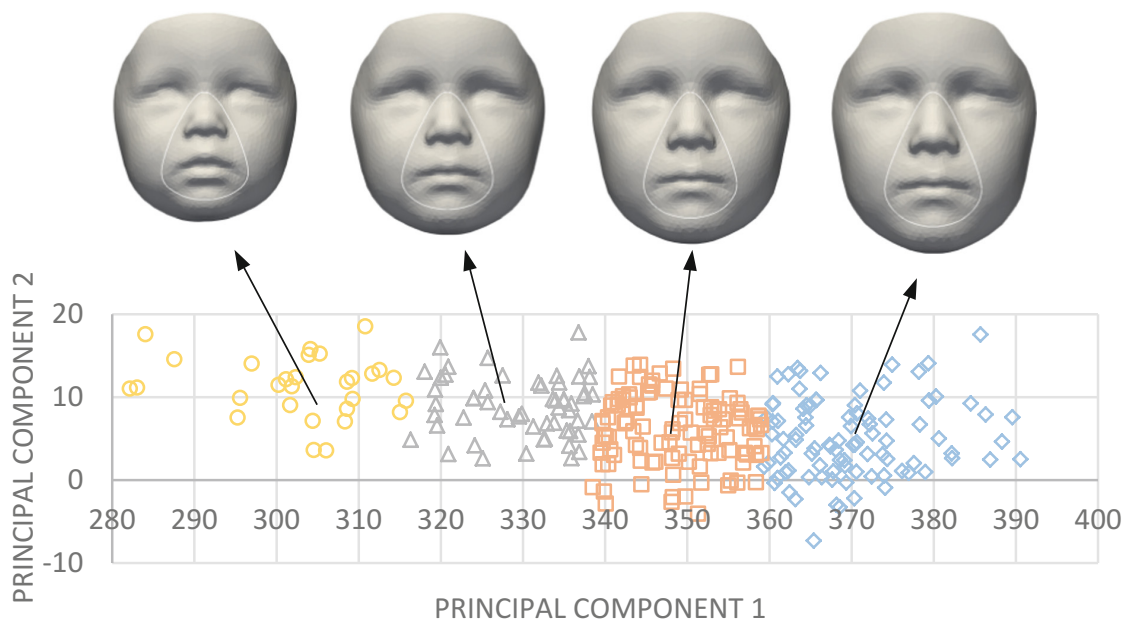

$\diamond$ Cluster $1 \triangle$ Cluster $2 \circ$ Cluster $3 \quad \square$ Cluster 4

Fig. 5. Scatterplot of key dimension based Principal Component 1 against Principal Component 2 divided into four clusters and the RFM's.

Table 2. Number of participants in each PCA based cluster.

\begin{tabular}{l|l|r}
\hline \multicolumn{3}{l}{$\begin{array}{l}\text { Number of cases in } \\
\text { each cluster }\end{array}$} \\
\hline Cluster & 1 & 92 \\
\cline { 2 - 3 } & 2 & 57 \\
\cline { 2 - 3 } & 3 & 29 \\
\cline { 2 - 3 } & 4 & 112 \\
\cline { 2 - 3 } & Total & 290 \\
\hline
\end{tabular}




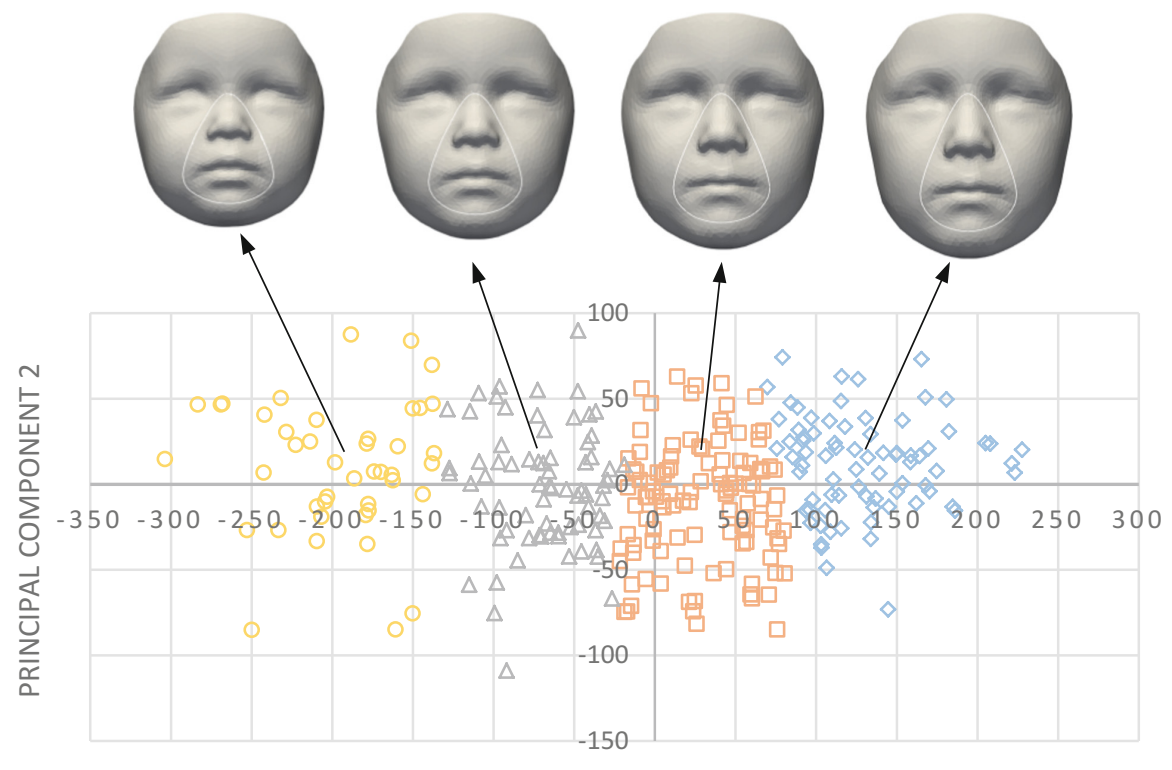

PRINCIPAL COMPONENT 1

\section{$\diamond$ Cluster $1 \square$ Cluster $2 \Delta$ Cluster $3 \circ$ Cluster 4}

Fig. 6. Scatterplot of shape based Principal Component 1 against Principal Component 2 divided into four clusters and the RFM's.

Table 3. Number of participants in each shape based PCA cluster.

\begin{tabular}{|c|c|c|}
\hline \multicolumn{3}{|c|}{$\begin{array}{l}\text { Number of cases in } \\
\text { each cluster }\end{array}$} \\
\hline \multirow{5}{*}{ Cluster } & 1 & 77 \\
\hline & 2 & 111 \\
\hline & 3 & 72 \\
\hline & 4 & 43 \\
\hline & Total & 303 \\
\hline
\end{tabular}

\subsection{Mean Distance to RFM Within Each Cluster for Each Method}

Figure 7 shows the results of the comparison between the RFM and the cluster members. Less variation (blue) was observed in the area surrounding the sellion landmark (nose bridge area) because the template registered faces were aligned with the origin point at that landmark. Variation was the highest in the regions surrounding the chellion landmarks for the smallest RFM of all three methods. 

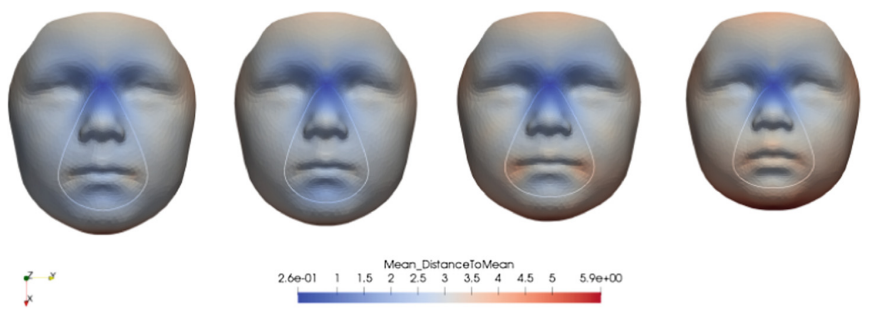

(a) Bivariate
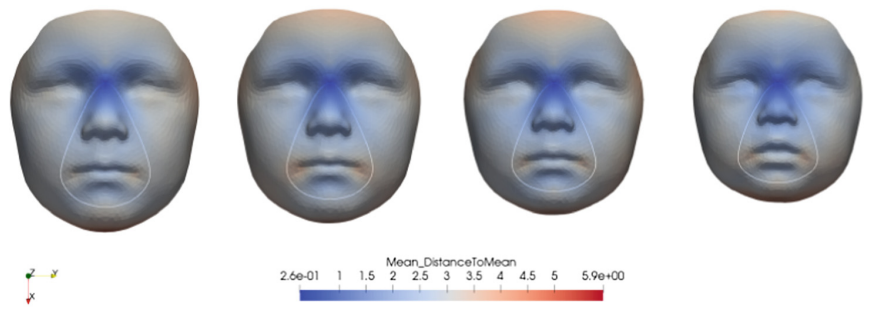

(b) PCA
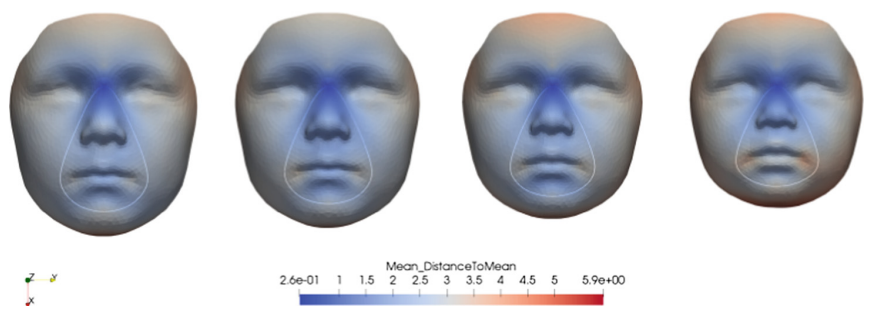

(c) Shape based PCA

Fig. 7. Colour map of the mean distance to RFM (mm) per cluster. High variability is indicated by red and low variability by blue. (Color figure online)

Table 4. Overview of the mean distance $(\mathrm{mm})$ between the vertices of the RFM and the vertices of individual faces over the face mask area per method and per cluster.

\begin{tabular}{l|l|l|l|l|l|l}
\hline \multicolumn{2}{c|}{} & \multicolumn{3}{|l|}{ Cluster } & \multirow{2}{*}{ Sum } \\
\cline { 3 - 7 } \multicolumn{2}{c|}{} & 1 & 2 & 3 & 4 & \\
\hline \multirow{3}{*}{ Method } & BIVARIATE & 2.35 & 2.35 & 2.54 & 2.74 & 9.98 \\
\cline { 2 - 7 } & PCA & 2.36 & 2.30 & 2.16 & 2.16 & 8.98 \\
\cline { 2 - 7 } & PCASHAPE & 2.10 & 1.93 & 1.98 & 2.15 & 8.16 \\
\hline
\end{tabular}

\section{Discussion}

Initial, visual inspection of the RFM's seem to indicate that for each cluster the RFM's are relatively similar to each other, regardless of the utilized method. When analysing the RFM's in further detail, small differences could be observed. For the multi-variate 
and shape based RFM's the overall shape and size changes gradually from smallest to largest. However, for the bivariate method there appears to be a jump in the width of the mouth from cluster 2 to 3, resulting in cluster 3 having a relatively larger mouth. This may be due to the fact that mouth width directly influences the RFM in the bivariate method whereas in both PCA's the first component described the variation in the overall size of the face and the second component was more related to the width of the face

The comparison of each RFM to their cluster members shows that the largest variations were observed in each of the smallest RFM's, regardless of method. This could be explained by the rapid growth phases that occur mostly in the first few years of a child's life [14] resulting in larger differences in the facial dimensions for children within these clusters. Interestingly, the mean inter-vertice distance of the RFM for each method (Table 4) shows that the more information is incorporated (i.e. more dimensions) in the analysis, the smaller the mean distance becomes. The mean distance is largest for the bivariate RFM and smallest for the shape based RFM's. This indicates that, for the latter, the shape based RFM's is more representative for it's respective cluster.

Firstly, it is important to note that the number of participants assigned to each cluster varied per method from $\mathrm{N}=2$ to $\mathrm{N}=13$, in part due to the method of clustering but also for practical reasons as described in previous research [8]. This often occurred in age groups one (aged 6 months to 1.5 years) and two (1.5 to 2.5 years). This will, to a certain extent, have influenced the generation of the PCA RFM's for the clusters that represent the smallest children.

Secondly, it is important to note, that in this study, the distribution was divided into four clusters. Determining the number of clusters in order to develop a sizing system depends on the type of data, the required fit, cost-benefit, etc. and is often a combination of statistical and practical experience $[2,13]$. Further research is needed in order to determine the ideal number of clusters (sizes) for the development of the mask or any other product for that matter.

Further study is needed in order to analyse the differences of the contours related to the RFM's in order to investigate the implications for the design of the mask or for the design of other products where fit is an important factor.

At present, sizing system development based on a bivariate distribution seems more intuitive for designers because there is a direct link between body dimensions and product dimensions. This approach is partially based on statistics but also practical knowledge (material properties, etc.). It is important to note however, that all three methods use shape data to develop models rather than the classical anthropometric approach whereby anthropometric measurements are directly translated to product dimensions.

Even though the Multivariate approach incorporates multiple product relevant dimensions, the outcome is not directly translatable to product dimensions. More statistical knowledge form the designers side is needed but the development of representative models offers a solution that can be incorporated in the design process. A solution would be to develop a software tool which allows for this approach without having detailed statistical knowledge. 


\section{References}

1. Dainoff M, Gordon C, Robinette K, Strauss M (2004) Guidelines for using Anthropometric data in product design - HFES 300 Commitee, Santa Monica

2. Hsiao H (2013) Anthropometric procedures for protective equipment sizing and design. Hum Factors 55:6-35. https://doi.org/10.1177/0018720812465640

3. Luximon A, Zhang Y, Luximon Y, Xiao M (2012) Sizing and grading for wearable products. CAD Comput Aided Des 44:77-84. https://doi.org/10.1016/j.cad.2011.07.004

4. Zhuang Z, Bradtmiller B, Shaffer RE (2007) New respirator fit test panels representing the current U.S. civilian work force. J Occup Environ Hyg 4:647-659

5. Ball RM (2011) SizeChina: a 3D Anthropometric survey of the Chinese head. Delft University of Technology

6. Ballester A, Valero M, Nacher B, et al (2015) 3D body databases of the Spanish population and its application to the apparel industry. In: Proceedings of 6th International Conference 3D Body Scanning Technol Lugano, Switzerland, 27-28 October 2015, pp 232-233. https:// doi.org/10.15221/15.232

7. Wuhrer S, Shu C, Bose P (2012) Automatically creating design models from 3D Anthropometry data. J Comput Inf Sci Eng 12:41007. https://doi.org/10.1115/1.4007839

8. Goto L, Lee W, Molenbroek JFM, et al (2017) Traditional and 3D scan extracted measurements of the heads and faces of Dutch children. Submitted

9. Lee W, Goto L, Molenbroek JFM, Goossens RHM (2017) Analysis methods of the variation of facial size and shape based on 3D face scan images. In: Proceedings of the human factors and ergonomics society October 2017, pp 1409-1413. https://doi.org/10.1177/ 1541931213601836

10. Amirav I, Luder AS, Halamish A, et al (2013) Design of aerosol face masks for children using computerized 3D face analysis 26:1-7 https://doi.org/10.1089/jamp.2013.1069

11. Goto L, Lee W, Molenbroek JFM, et al (2018) Analysis of a 3D Anthropometric Data Set of Heads and Faces of Children for Design Applications. submitted

12. Niu J, Li Z, Salvendy G (2009) Multi-resolution shape description and clustering of threedimensional head data. Ergonomics 52:251-269. https://doi.org/10.1080/ 00140130802334561

13. Lacko D, Huysmans T, Vleugels J et al (2017) Product sizing with 3D anthropometry and kmedoids clustering. CAD Comput Aided Des 91:60-74. https://doi.org/10.1016/j.cad.2017. 06.004

14. Farkas LG, Posnick JC, Hreczko TM (1992) Growth patterns of the face: a morphometric study. Cleft Palate-Craniofacial J 29:308-314. https://doi.org/10.1597/1545-1569(1992) 029\%3c0308:gpotfa\%3e2.3.co;2 